\title{
Física del caos en la predicción meteorológica
}

https://doi.org/10.31978/639-19-010-0.755

\author{
Carlos Santos Burguete ${ }^{1}$ (csantosb@aemet.es)
}

${ }^{1}$ AEMET / Dirección de Producción e Infraestructuras / Centro Nacional de Predicción

\begin{abstract}
RESUMEN
Un amplio elenco de expertos en modelización del tiempo y el clima, predicción numérica y operativa, así como una serie de áreas afines se reunieron en 2017-2018 para escribir un compendio de conocimiento titulado «Física del caos en la predicción meteorológica», coordinado por el autor y cuya edición corría a cargo de AEMET. Se presenta aquí el libro en su conjunto, a modo de resumen, haciendo hincapié en su carácter integrador, con la diversidad de aspectos abordados en el mismo: historia, fundamentos, sistemas, aplicaciones, casos de estudio, sociedad, etc. Este artículo se compone básicamente de extractos del libro adecuadamente seleccionados correspondiendo, de forma casi biunívoca, cada sección del artículo con cada parte del libro.
\end{abstract}

PALABRAS CLAVE: sistemas de predicción por conjuntos; predicción probabilista; predicción operativa; modelización; meteorología y sociedad; fenómenos meteorológicos adversos.

\section{INTRODUCCIÓN}

Esta obra integradora partía con varios objetivos diferentes: en primer lugar, cubrir un importante hueco bibliográfico en castellano: en 2017 no había monográficos completos ni profundos que describieran los sistemas de predicción por conjuntos (SPC) ni la predicción probabilista. Se deja así el legado de una experiencia profesional en la Agencia Estatal de Meteorología (AEMET), colaborando con numerosas entidades nacionales, extranjeras o internacionales, tales como centros de investigación y desarrollo, centro operativos, universidades, etc. Se intenta poner al alcance de un amplio abanico de lectores este compendio de conocimiento, con la idea de ofrecer principalmente un libro de consulta para profesionales de la meteorología, pero sin renunciar a servir también como introducción amena para no iniciados en el mundo de la predicción y la predecibilidad.

Han contribuido al libro más de 100 autores, expertos importantes en sus especialidades en España y otros lugares del mundo. Más de 20 fotógrafos, profesionales o amateurs, así como más de 10 retratistas ilustran el libro, en cabeceras de parte o capítulo, cuadros con reseñas de personalidades, etc. (fíg. 1). El libro consta de más de 1200 páginas, más de 60 capítulos (sin contar anexos o memorias) y casi 700 entradas bibliográficas. Participaron Argentina, México, Portugal, Austria, Hungría, Alemania; los centros ECMWF, HIRLAM, CRAHI, IPMA, UKMO, ZAMG, CSIC, RTVE, DLR, entre otros; y las universidades UIB, UCM, UAM, UPM, U. Constanz.

Preceden, a la parte propia de introducción, dos prólogos de dos grandes figuras de la predecibilidad y la predicción, respectivamente: EugENIA KALNAY, de la Universidad de Maryland y, anteriormente, directora de investigación en el NCEP, pionera mundial en el desarrollo de SPC y ÁNGEL RIVERA, excelente profesional de la predicción en AEMET (anteriormente INM) y excelente divulgador de la meteorología.

Para introducir el tema de forma sintética, se recoge acto seguido la página de presentación del libro. La predicción del tiempo y del clima despierta un interés creciente en la sociedad, especialmente los 
a)

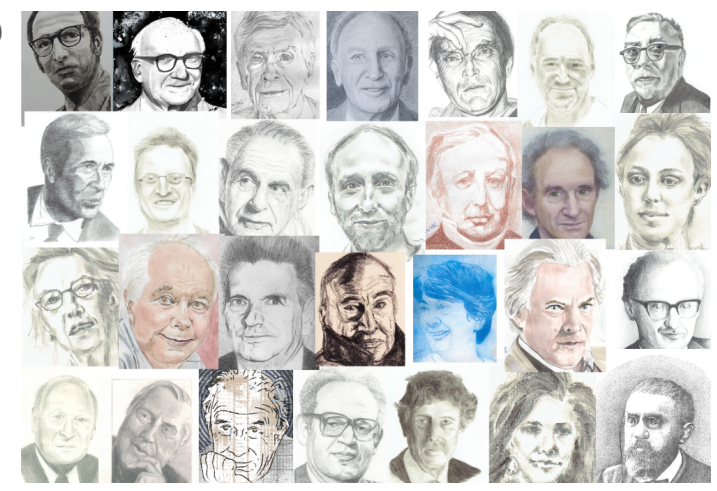

b)

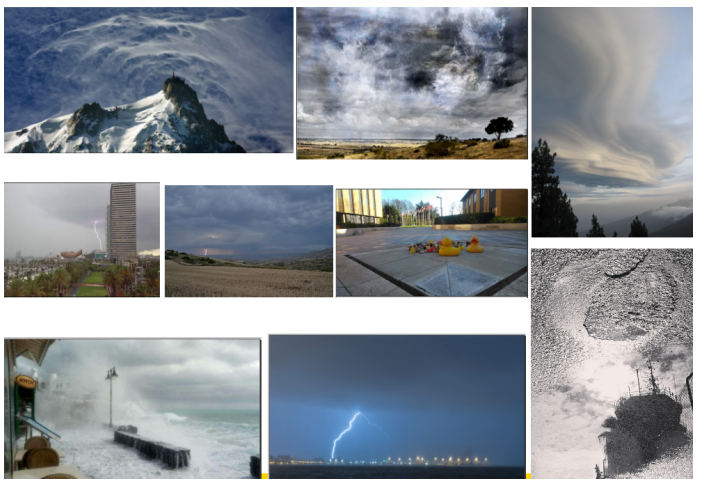

c)

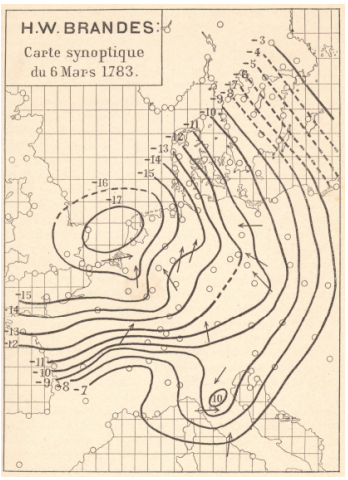

d)

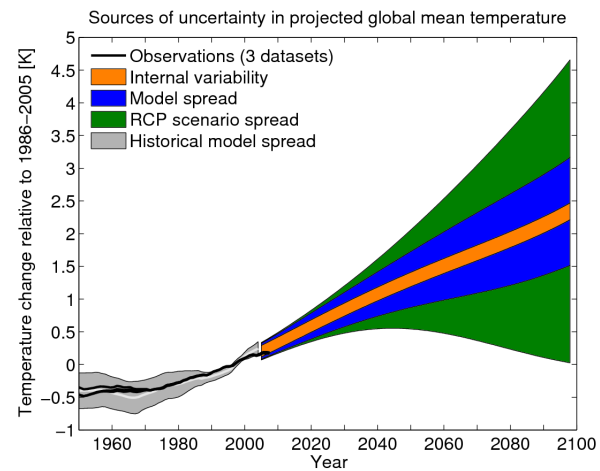

Figura 1.

a) Algunos retratos de los que ilustran el libro; b) ídem con fotografías; c) primer mapa sinóptico de la historia, HildebrandsSON-BRANDES; d) Fuentes de incertidumbre en la simulación climática (CUBASCH et al., 2013).

fenómenos adversos y el calentamiento global. ¿Hasta cuántos días son fiables las predicciones del tiempo? ¿En qué se basa la predicción por localidades? ¿Pueden hacerse predicciones con seis meses de antelación? ¿Cómo puede predecirse el cambio climático? ¿Se harán predicciones cada vez más exactas? ¿Qué es el efecto mariposa? Intentando dar respuesta a estas preguntas, este libro aproxima al lector al mundo de la meteorología y, en particular de la predicción del tiempo, que se apoya enormemente en los modelos atmosféricos, en los que se ha introducido en las últimas décadas la física del caos para conseguir resultados más realistas. Manteniendo una visión general divulgativa se ofrece una visión ecléctica de la meteorología y la predicción, intentando abordar equilibradamente diferentes aspectos, empezando por una breve historia de la predicción y de la física del caos. Manteniendo también rigor científico y técnico, se introducen e ilustran tanto el problema de la predecibilidad, como los fundamentos de los sistemas de predicción por conjuntos. Entre sus numerosas aplicaciones mostramos algunas, como la predicción aeronáutica, del oleaje o de rayos, los medicanes, la meteorología en el Mediterráneo y, con especial énfasis, las predicciones mensual y estacional, así como las proyecciones climáticas, tocando de lleno el problema del calentamiento global y el cambio climático. La aplicación a la predicción basada en probabilidades se ejemplifica con una serie de casos de estudio, tanto de fenómenos meteorológicos adversos como predicciones cotidianas. A los aspectos sociales, de capital importancia, se dedica una parte específica que incluye, entre otros, la percepción de la meteorología y la probabilidad, así como el reto de la comunicación en los medios de la predicción probabilista.

La primera parte del libro, precisamente dedicada a introducir los aspectos más básicos, arranca con un capítulo a modo de introducción general, con las cuestiones genéricas que pueden motivar la exposición de diferentes líneas de investigación y resultados que se van a ir mostrando a lo largo de la obra. Los tres capítulos que siguen son preparaciones imprescindibles para distintos aspectos abordados en el libro: una introducción histórica a la física del caos, una perspectiva también histórica de la predicción del tiempo y el advenimiento de una base científica para la misma y, por último, un acercamiento a la denominada predicción operativa, para dar a conocer la profesión de los predictores del tiempo y las diferentes dificultades a las que deben enfrentarse en el día a día.

El libro está dedicado a la memoria de Antonio Mestre Barceló, Rafael Ancell Trueba y Óscar García Colombo. 


\section{FUNDAMENTOS}

Dedicamos una parte importante del libro a los fundamentos científicos y técnicos de la predicción y la observación del tiempo, así como de la física del caos y su incursión en la modelización numérica de la atmósfera. Empezamos por una exposición sencilla de la física del caos, seguida de un modesto pero audaz resumen de las ciencias de la atmósfera. Abordamos después la observación meteorológica: un capítulo para los satélites, otro para el radar y redes de rayos y un tercero para observaciones de diversos tipos en tierra, con un breve resumen de la climatología como ciencia y técnica. Sigue una pareja importante de capítulos dedicados, respectivamente, a los modelos atmosféricos y a la supercomputación, con la problemática teórica y práctica, respectivamente, de la simulación de un sistema tan complejo como la atmósfera. Las limitaciones de los modelos deterministas se exponen en el capítulo de predecibilidad y, en el siguiente, se presenta a los protagonistas del libro: los sistema(s) de predicción por conjuntos (SPC), integrando el caos en la predicción numérica y dando pronósticos puramente probabilistas. El postproceso estadístico de estos sistemas puede mejorar sus predicciones, lo que se cuenta en el capítulo siguiente. Cierra esta parte el capítulo dedicado a la verificación de estos SPC: cómo evaluar sus resultados.

a)

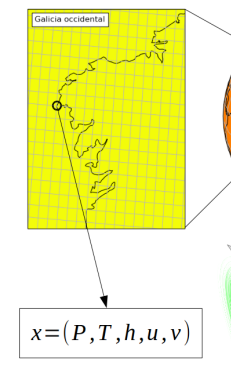

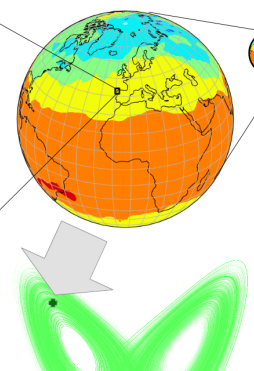

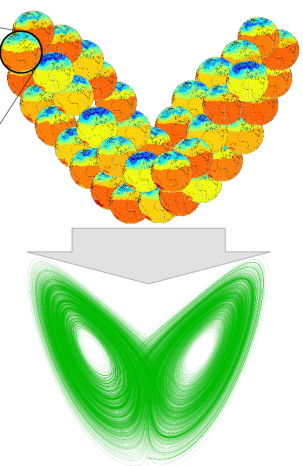

b)

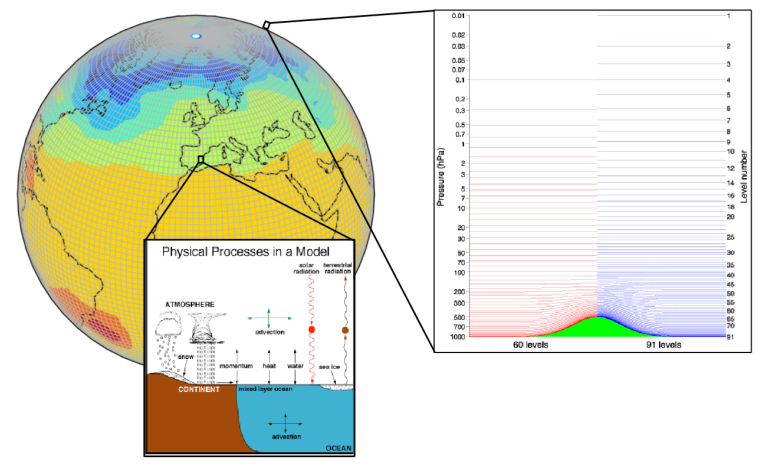

c)

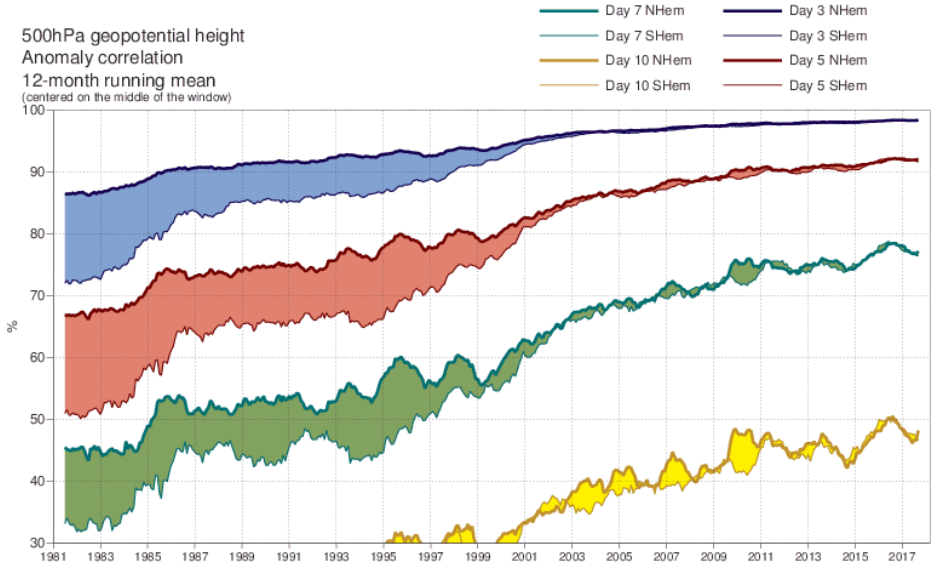

Figura 2.

a) Atractor de Lorenz como símbolo de los estados atmosféricos;

b) esquema de un modelo atmosférico; c) mejoras en el rendimiento del modelo del Centro Europeo de Previsiones a Medio Plazo (ECMWF, de sus siglas en inglés) a través de los años.

\section{DISEÑO}

Debido su tremenda complejidad e importancia, dedicamos una parte independiente del libro al diseño de los SPC, dividida en tres capítulos. Cada uno expone las técnicas de diseño específicas para el muestreo de alguno de los tres tipos correspondientes de errores e incertidumbres: en las condiciones iniciales, en la formulación del propio modelo y, por último, en las condiciones de contorno. Este último tiene sentido solo en aquellos sistemas compuestos por modelos de área limitada, que son precisamente aquellos que AEMET desarrolla. 
a)

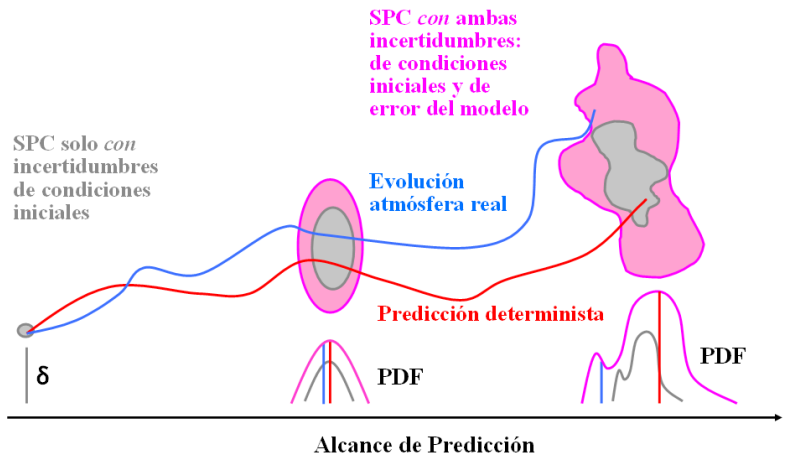

c)

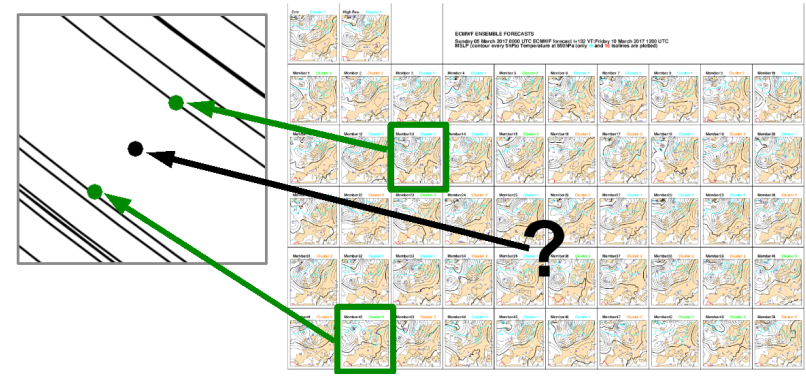

b)

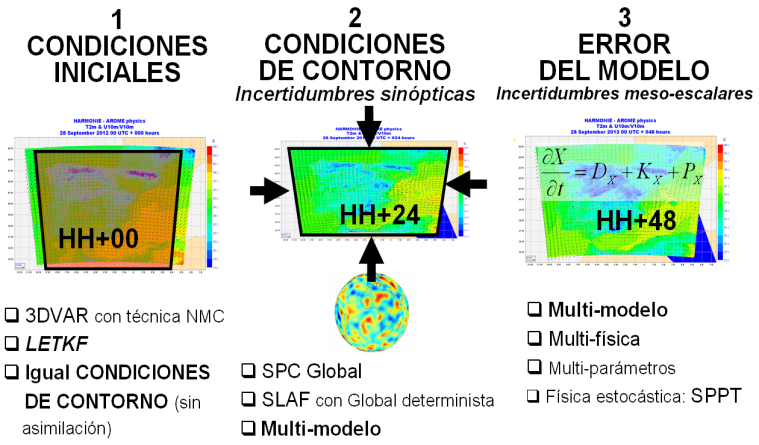

d)

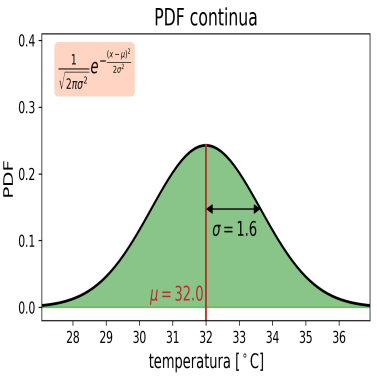

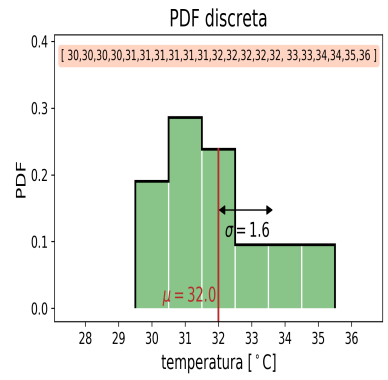

Figura 3. a) Tratamiento teórico de las incertidumbres en los SPC; b) implementación técnica del muestreo de las diferentes incertidumbres en los SPC; c) ilustración de la idea de que el promedio de un SPC puede no ser una situación atmosférica plausible; d) predicción probabilista, PDF continua y discreta.

\section{SISTEMAS}

A lo largo y ancho del mundo, diferentes centros de modelización numérica de la atmósfera y el océano han desarrollado distintos SPC. Al principio, solo los centros principales del mundo y, poco a poco, han seguido otros. Sabiendo que es imposible describir todos ellos con el detalle que merecen, hemos seleccionado los que conocemos mejor, por trabajar directa o indirectamente en su desarrollo o por utilizarlos como referencia o como herramienta. Comenzamos en la escala global con un capítulo dedicado al SPC global llamado ECENS, del European Centre for Medium-range Weather Forecasts - Centro Europeo de Predicción a Plazo Medio-(ECMWF). Pasando a la escala regional europea, describimos el GLAMEPS de HIRLAM en otro capítulo. Siguen dos capítulos para mostrar los sistemas hechos en España: AEMET-SREPS y el reciente AEMET- $\gamma$ SREPS. Los sistemas del Reino Unido y de México se describen después. Acto seguido, exponemos el proyecto MyWave de predicción del oleaje por conjuntos. Cerramos esta parte con un capítulo que intenta describir, de forma muy general, una buena parte de los SPC del mundo, tomando como marco de referencia los proyectos The (Thorpex) International Grand Global Ensemble (TIGGE) y TIGGE-LAM.

a)

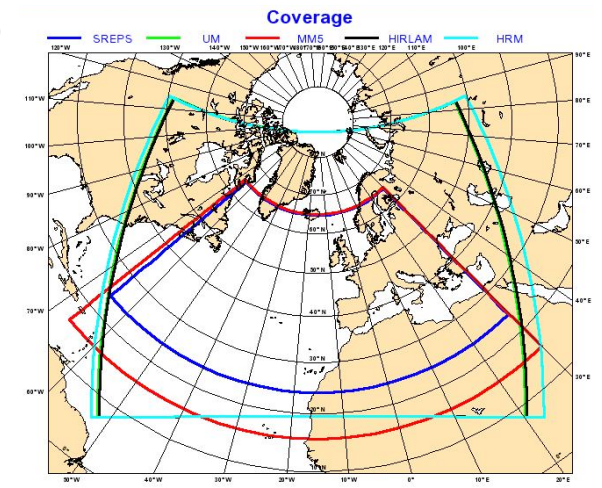

b)

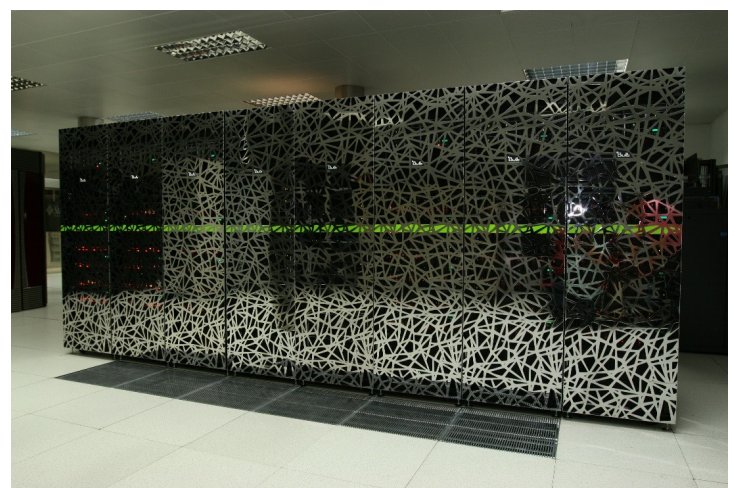

Figura 4. a) Dominios de los diferentes modelos integrantes del SPC AEMET-SREPS; b) superordenador Bull en la actualidad en AEMET. 


\section{APLICACIONES}

Incluimos en esta parte un abanico amplio y heterogéneo de diversas aplicaciones de los SPC. La naturaleza de cada capítulo puede pedir un enfoque más bien teórico o relativamente práctico. Para dar soporte teórico a toda la parte de Casos, es imprescindible abrir esta parte de Aplicaciones con un capítulo amplio dedicado a la predicción probabilista como tal: cómo abordar la predicción del tiempo usando estos SPC. Seguimos con exposiciones bien detalladas y rigurosas, con un capítulo de predicciones de largo plazo (mensual, estacional y anual) y otro para proyecciones del clima, que aborda las candentes cuestiones del calentamiento global y el cambio climático. Un capítulo breve cuenta después cómo pueden usarse observaciones de radar de forma probabilista para diseñar productos muy útiles, aplicables por ejemplo en el llamado nowcasting. De crucial importancia es la predicción aeronáutica, en la que pueden aplicarse con extrema utilidad los SPC de escala convectiva. Siguen dos capítulos específicos del Mediterráneo, uno de propósito general y otro sobre previsión de medicanes, los huracanes del Mare Nostrum. En relación con los avisos y los fenómenos adversos, presentamos en dos capítulos la previsión de rayos y una aplicación específica para los fenómenos meteorológicos adversos. Aunque nos gustaría mostrar aplicaciones de los SPC en muchos lugares del mundo, hemos elegido dos por cercanía cultural o linguística: Portugal y Argentina. Finalizamos esta parte con dos capítulos que muestran cómo pueden ser útiles y rentables las predicciones probabilistas en la optimización de las energías sostenibles: uno para la solar y otro para la eólica.

a) HARMONIE: Rayos estimados ( $\mathrm{rayos} / \mathrm{km}^{2}$ ). Pas: 29-08-2017 00Z Intearacion 29-08-2017 00 - 30-08-2017 00Z

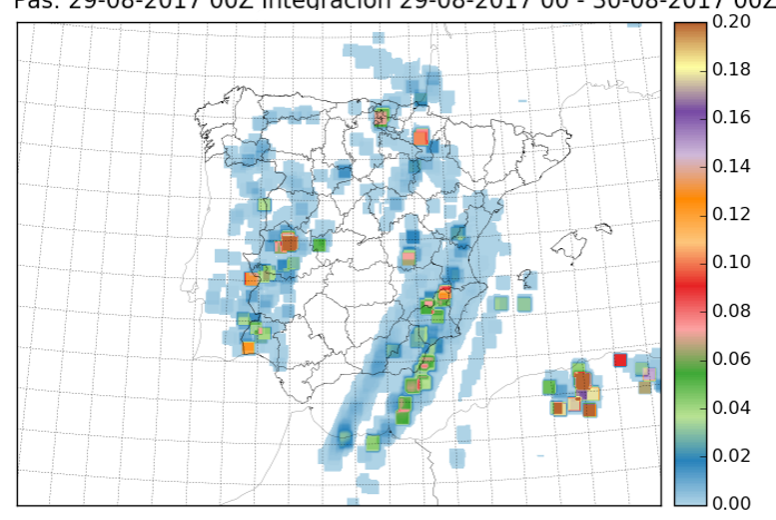

c)

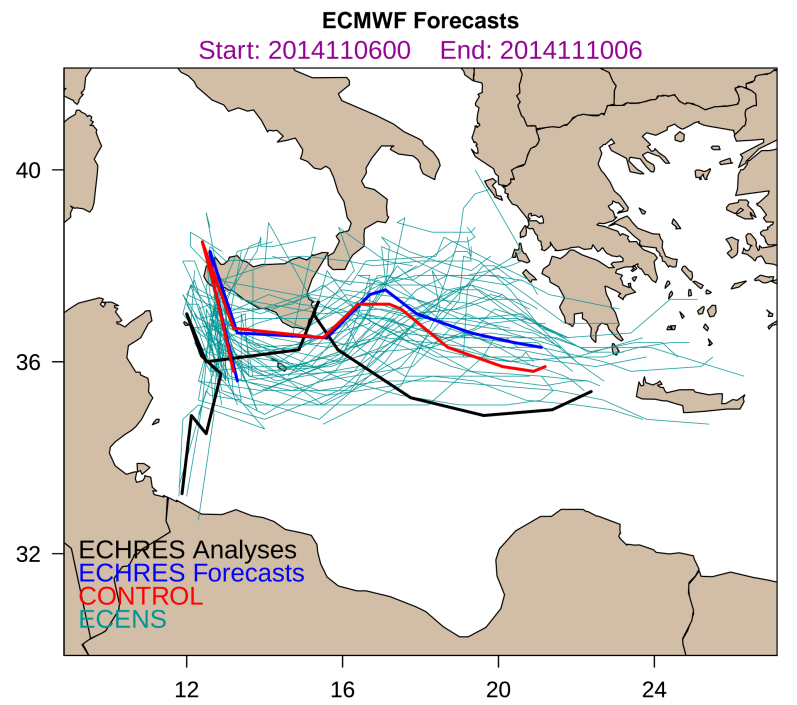

(a) b)

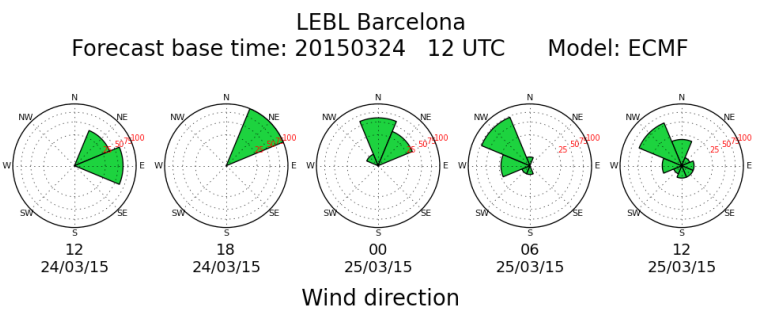

d)

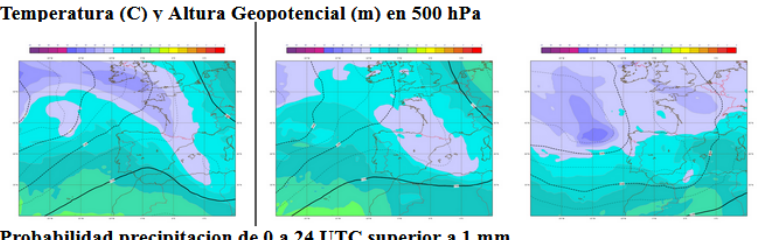

Probabilidad precipitacion de 0 a 24 UTC superior a $1 \mathrm{~mm}$

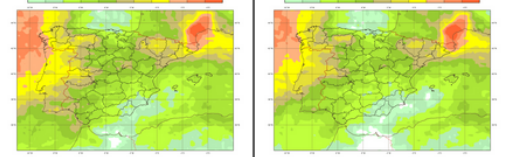

Probabilidad precipitacion de 0 a 24 UTC superior a $2 \mathrm{~mm}$

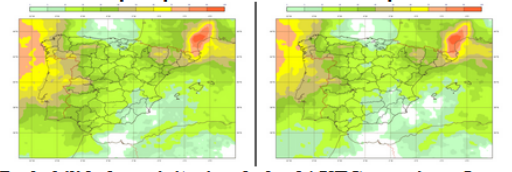

Probabilidad precipitacion de 0 a 24 UTC superior a $5 \mathrm{~mm}$
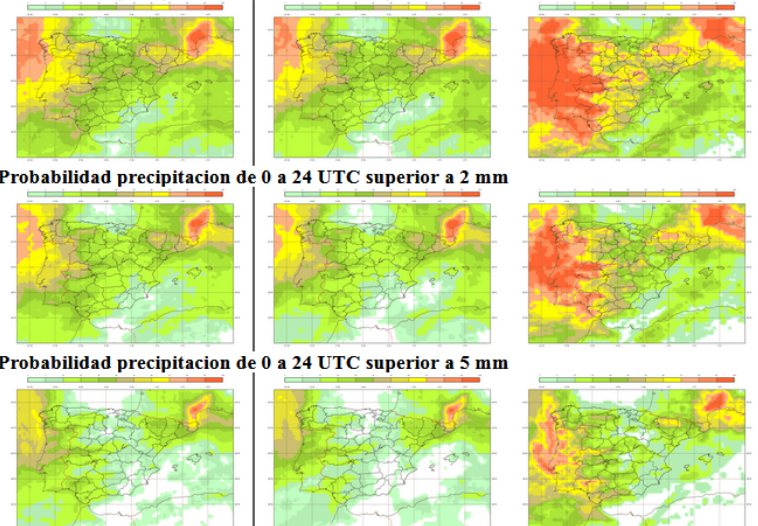

Probabilidad precipitacion de 0 a 24 UTC superior a $10 \mathrm{~mm}$
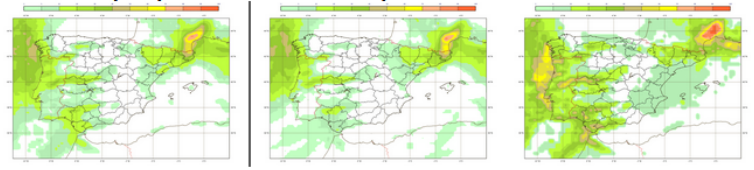

Figura 5. a) Previsión probabilista de densidad de rayos; b) EPSgrama aeronáutico de direcciones de viento en el aeropuerto de Barcelona; c) previsión probabilista de mediterranean hurricanes (huracanes mediterráneos) o medicanes; d) agrupamiento de escenarios en predicción probabilista. 


\section{SOCIEDAD}

Las predicciones probabilistas, en fondo y forma, son enormemente útiles para la sociedad. La psique humana entiende los conceptos relacionados con la probabilidad de modos diversos y con sesgos cognitivos que es conveniente conocer. No es una cuestión trivial presentar las predicciones al público usando lenguaje probabilista: es un auténtico reto para los medios. Estos aspectos se abordan con mucho detalle y profundidad en un capítulo desarrollado por profesionales de la psicología/antropología y de los medios de comunicación. El sistema europeo Meteoalerta de avisos de fenómenos meteorológicos adversos, conocido ampliamente por estar presente en los medios (avisos amarillo-naranja-rojo), se presenta en otro capítulo. En el capítulo siguiente tenemos oportunidad de mostrar el proyecto europeo Bridging of Probabilistic Forecasts and Civil Protection (PROFORCE), para la aplicación de los SPC a la emisión de avisos meteorológicos. Cerramos esta parte con un capítulo relacionado con la utilización del lenguaje meteorológico en los medios y en la sociedad, haciendo hincapié en la medida en que puede mejorarse la comunicación de la información meteorológica si se utiliza ese lenguaje con propiedad.

a)

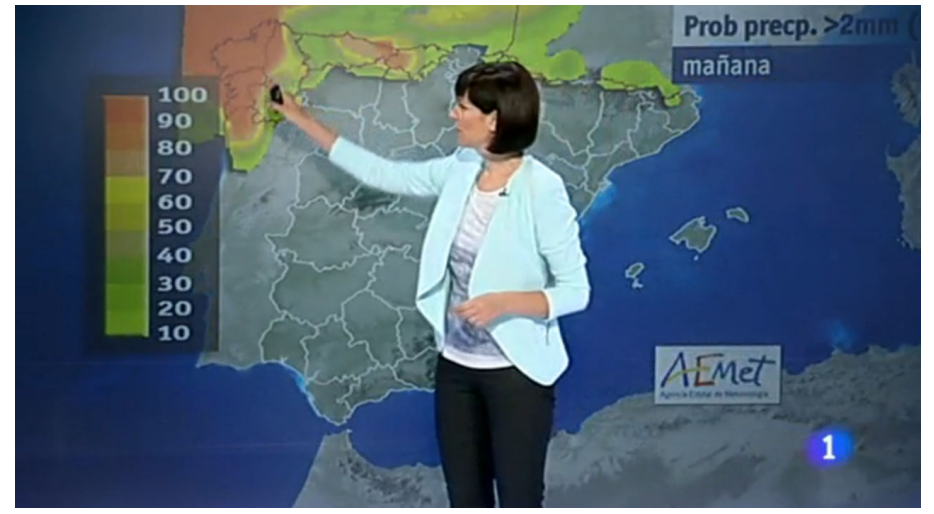

b)

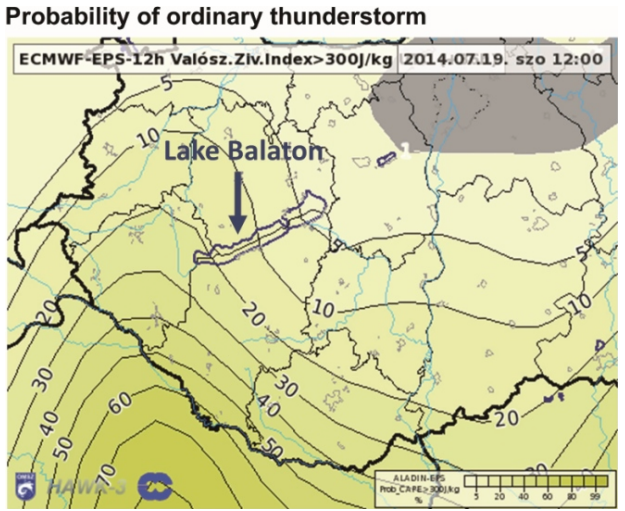

Figura 6. a) Mónica López presentando un mapa de probabilidad de precipitación en RTVE; b) mapa de probabilidad de tormenta ordinaria en Europa central dentro del proyecto PROFORCE.

\section{CASOS}

Como parte de la meteorología, la predicción del tiempo tiene una naturaleza variada, pues es ciencia, es también técnica y, así mismo, un poco arte. Así, estamos aún lejos de poder automatizar el proceso de predicción (aunque haya algunos que lo piensen). Los casos de estudio, a menudo fenómenos espectaculares o, por lo menos, extremos y, otras veces, más bien cotidianos, son fundamentales en el aprendizaje y en la exposición de la meteorología y, en particular, de la predicción y la observación del tiempo. Un selecto elenco de predictores operativos o afines, con renombrada experiencia, exponen en esta parte algunos de estos casos de estudio. Empezamos con un caso histórico, además de espectacular: el récord de Oliva-Gandía, con una precipitación acumulada de $817 \mathrm{~mm}$ registrados en 24 horas. Entre el resto de los casos mostramos predicción de aludes, un tema escasamente abordado en la literatura meteorológica. Por supuesto, exponemos después casos de vientos y/o precipitaciones intensos, lluvias torrenciales, nevadas, inundaciones, olas de calor, sin olvidarnos de los huracanes. Estos últimos, aunque no son casos acaecidos en España, los exponemos por el enorme interés que presentan. Del mismo modo presentamos una predicción convencional en Myanmar. Se muestran también algunos casos de predicciones críticas por su impacto social, por ejemplo Semana Santa de 2017. Algunos casos son interesantes por su escasa predecibilidad, uno de los conceptos clave en este libro. 
a)

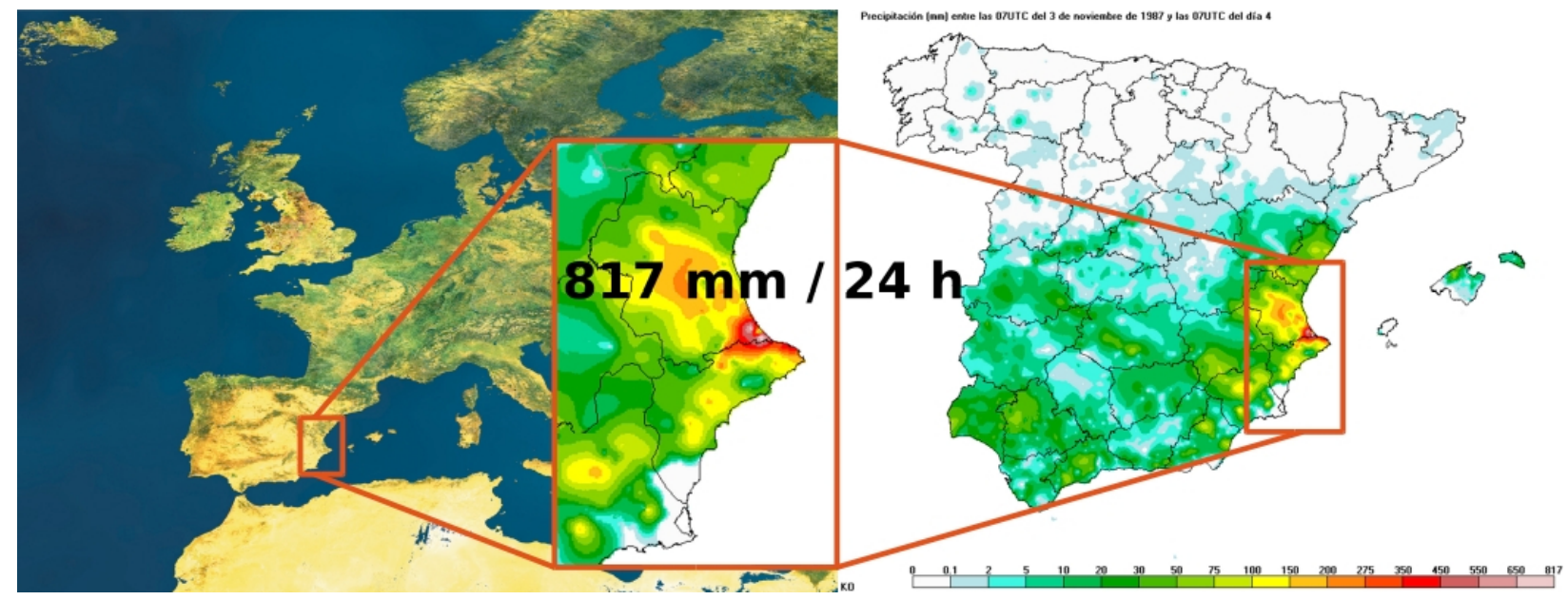

b)

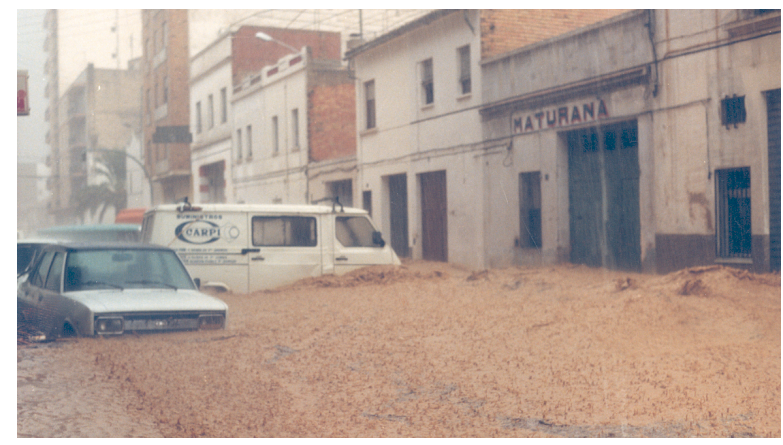

c)

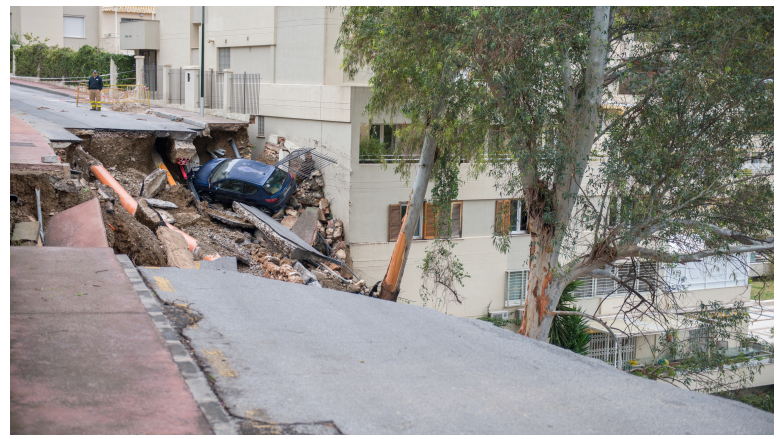

Figura 7. a) Récord nacional de precipitación en 24 h en Oliva-Gandía, 3-4 noviembre de 1987; b) impactos en el caso de Oliva; c) impactos del caso de febrero de 2017 en Málaga.

\section{CONCLUSIONES}

Resumimos, en el único capítulo que comprende esta parte, las ideas principales desarrolladas en el libro. Respondemos, así mismo, a las preguntas con las que abríamos en la introducción, preguntas que la sociedad formula a la comunidad meteorológica. El camino para ofrecer respuestas, en diversos niveles de profundidad, seguido en este libro, esperamos que sea en sí mismo esclarecedor para muchos. Exponemos también algunas de las principales preocupaciones de los profesionales de la meteorología y, en particular, de la predicción en relación con el futuro de estas disciplinas de ámbito científico y técnico. Esperamos, de corazón, haber dado algunas respuestas o, por lo menos, haber ampliado el abanico de preguntas con cuestiones motivadoras.

Hay una serie de aspectos fundamentales que podemos destacar de los SPC, algunos de ellos relacionados entre sí:

- Podemos simular el efecto de las diversas fuentes de error en la predicción.

- Nos permiten estimar la PDF de los estados de predicción.

- Proporcionan diferentes escenarios atmosféricos futuros y permiten al usuario estimar la probabilidad de esos escenarios.

- Nos dan información explícita, cuantitativa y detallada sobre la dispersión atmosférica, que está muy relacionada con la incertidumbre y, por ende, con la predecibilidad.

- Permiten identificar áreas y/o alcances predictivos con baja predecibilidad. 
- La predecibilidad es dependiente del flujo. Podemos, con los SPC, calibrar esa dependencia, encontrando relaciones entre la predecibilidad y los patrones atmosféricos (distintas situaciones) con los que trabajamos.

- Nos permiten evaluar el riesgo potencial de fenómenos adversos. Predecir la predecibilidad es predecir el riesgo meteorológico y climático, un elemento esencial en cualquier sistema práctico cuantitativo de predicción, especialmente en lo referente a estos fenómenos adversos.

- El promedio del ensemble no es necesariamente una situación meteorológica, ni debe ser tomado como escenario atmosférico plausible.

- Los productos para la predicción que proveen los SPC representan su carácter inherentemente probabilista.

- Los SPC se empezaron a utilizar en predicción operativa en AEMET durante los años 90 y son una poderosa herramienta, tanto para la predicción a escala sinóptica como, recientemente con el desarrollo de SPC kilométricos, para la predicción a mesoescala.

Se dan respuestas relativamente sintéticas a las preguntas del principio del libro, fundamentadas en los contenidos desplegados en el mismo: ¿Hasta cuántos días son fiables las predicciones del tiempo? A la luz de los conceptos alumbrados en el libro, ya sabemos que la mejor respuesta es «depende». ¿En qué se basa la predicción por localidades? ¿Pueden hacerse predicciones con seis meses de antelación? ¿Cómo puede predecirse el cambio climático? ¿Se harán predicciones cada vez más exactas y precisas? ¿Se usa la teoría del caos en la predicción del tiempo? ¿Qué es el efecto mariposa? ¿Si tuviéramos condiciones iniciales perfectas podríamos evitar el problema del caos determinista y traspasar el límite de predecibilidad de la atmósfera? ¿Pero en un futuro se podrá traspasar el límite de predecibilidad, verdad?

Se detallan conclusiones de la experiencia en el desarrollo de SPC en los siguientes aspectos:

- Multimodelo y multianálisis frente a un solo modelo.

- Impacto del tamaño del ensemble o número de miembros.

- Papel de la resolución.

- Predecibilidad de la convección.

Se describe el posible alcance del libro como sigue. Si los recursos en investigación acompañan a los, casi seguros, avances tecnológicos en satélites y en supercomputación, entonces el proceso de predicción del tiempo y proyección del clima mejorará en todas las escalas, llegando a operar en escalas cada vez más finas. Dentro de esas mejoras, aunque es de esperar una reducción en los errores y las incertidumbres, siempre estará presente un límite en la predecibilidad. Por tanto, todos los conceptos, metodologías y técnicas presentados en este libro seguirán siendo válidos en el futuro y útiles para las próximas generaciones. Así, algunas de las direcciones del futuro ya están trazadas.

\section{ANEXOS}

Algunos aspectos que pueden complementar los contenidos expuestos en las distintas partes del libro, se abordan ahora en estos anexos, que esperamos sean de interés, al menos tanto como las partes principales. Una introducción a la probabilidad no podía faltar en este volumen y, de hecho, hemos dudado mucho en integrarla en la parte de fundamentos, que ya estaba muy abultada. Las orografías de los principales modelos y SPC aparecidos en el libro se enseñan en una serie de mapas muy detallados, que pueden engrosar la lista de referencias básicas de la predicción operativa o ilustrar a los no iniciados en la dificultad de los modelos 
para «ver» muchos fenómenos de influencia orográfica. En el cine y la literatura encontramos numerosos casos en los que el caos o los fenómenos meteorológicos adversos, sin olvidar el cambio climático, son protagonistas; no hemos querido dejar pasar la ocasión de hacer una modesta reseña para algunas películas o novelas seleccionadas en un anexo. La terminología particular derivada de los SPC ocupa otro anexo. Dando un toque artístico precioso para el libro, algunos capítulos presentaban pequeños retratos de personajes, históricos o actuales, importantes en algunos de los aspectos abordados; todos esos retratos se recopilan, a página completa, en un anexo que cierra esta parte.

Orografía - Puntos de Grid - HARMONIE-AROME - 2017

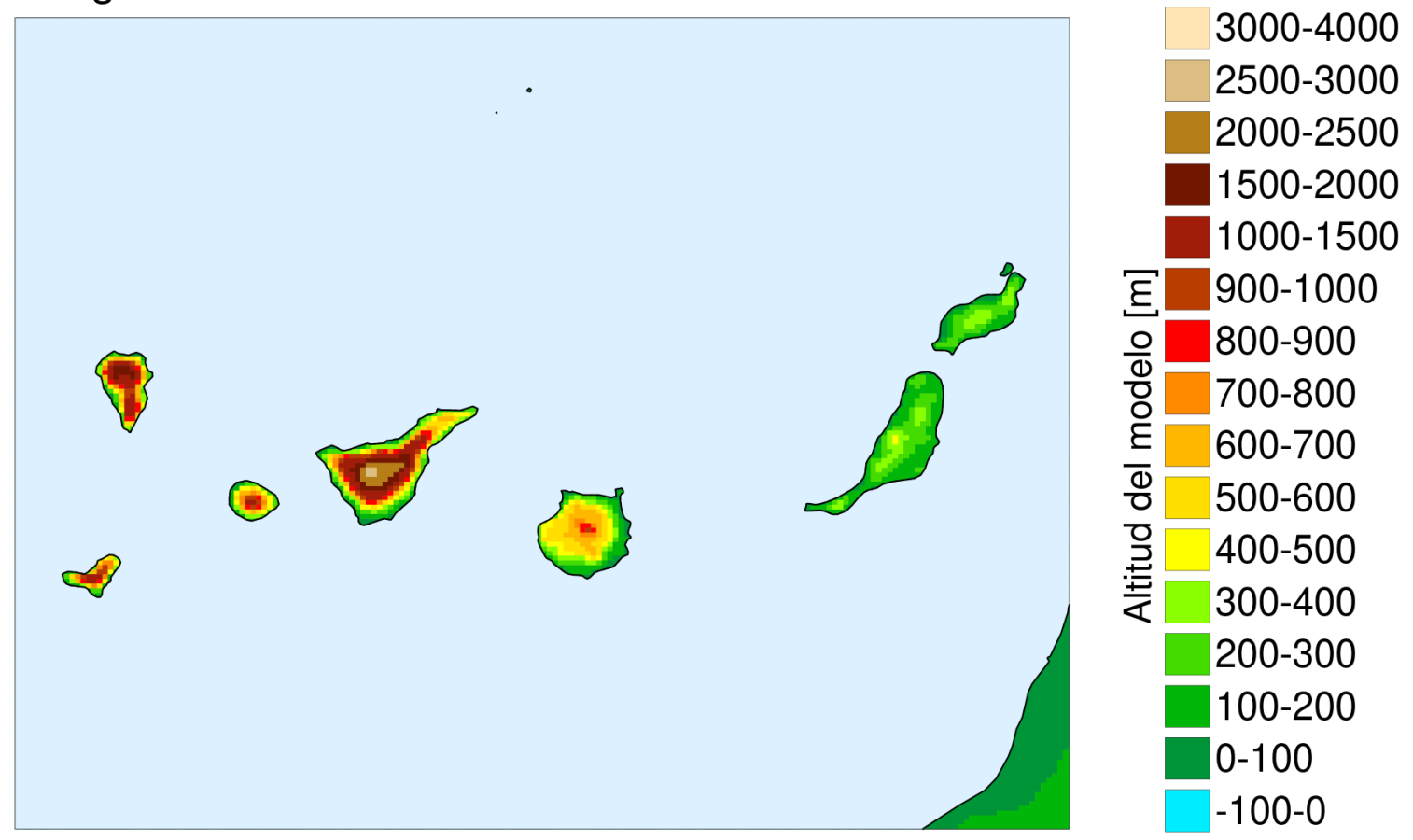

Figura 8. Orografía del modelo HARMONIE-AROME en Canarias.

\section{MEMORIAS}

Anunciábamos en el prefacio que dedicamos este libro a tres compañeros de AEMET que estuvieron involucrados, directa o indirectamente, en trabajos de investigación y desarrollo relacionados con los SPC. Estos colegas, tristemente, ya no están con nosotros. ANTONIO MeStre BARCELó, meteorólogo todoterreno, experto en climatología y predicción estadística, participó en la introducción de la predicción probabilista en AEMET y estuvo presente en una primera reunión del Grupo de Predecibilidad con EugENIA KALNAY. RAFAEL ANCELL TRUEBA, meteorólogo cántabro y amigo, colaborador activo con la Universidad de Cantabria, experto en análisis estadístico, climatología y predicción, colaboró también con el Grupo de Predecibilidad. ÓsCAR GARCíA Colombo, «Cuco», mente privilegiada, amigo del alma, experto en supercomputación, predicción y modelos, participó un tiempo en el Grupo de Predecibilidad. Como memoria a estas tres personas, hemos recuperado textos escritos o protagonizados por ellos para componer tres capítulos. Amigos, un abrazo desde este lado.

\section{BIBLIOGRAFÍA}

El libro incluye 685 referencias bibliográficas, organizadas en referencias, por un lado (la casi totalidad de entradas) y lecturas recomendadas, por otro. 


\section{2. ÍNDICES}

Cierra el libro una parte de índices diversos. Un índice alfabético resulta imprescindible en un libro de estas dimensiones. Añadimos una lista de acrónimos, nunca todo lo completa que debería ser, pero esperamos que suficiente. Terminamos con un glosario, tampoco todo lo completo que desearíamos, pero al menos resultará útil a la hora de consultar el libro y buscar algunos términos con rapidez. Para la elaboración de algunos términos fueron útiles los glosarios de AEMET y la American Meteorological Society (AMS).

\section{AGRADECIMIENTOS}

El libro es fruto de casi dos décadas de investigación, desarrollo y operación en ramas emergentes de la meteorología y la predicción como son la predecibilidad y los sistemas de predicción por conjuntos. Los diversos autores de este libro, tanto de AEMET como de otras instituciones forman, o han formado, parte de grupos de investigación, desarrollo o predicción relacionados con estos temas, así como la divulgación de los mismos. El diseño, desarrollo, puesta en operación y explotación, así como el uso en predicción de estos sistemas de predicción por conjuntos suponen un desafío científico y técnico de envergadura considerable, e incluyen el trabajo y apoyo de mucha gente, tanto de dentro como de fuera de AEMET. Ya sea brindando soporte y apoyo técnico, como conocimiento en discusiones de enorme valor científico, sin olvidar el material proporcionado expresamente para este libro.

La lista de agradecimientos es enorme y no puede resumirse sin engordar este artículo de resumen.

Tanto la edición del libro como la confección expresa de figuras se han realizado enteramente con software libre https://es.wikipedia.org/wiki/Software_libre.En particular y, sin entrar en demasiado detalle, trabajando sobre sistema operativo Linux Ubuntu, la edición se hizo con Lyx+LaTeX https://www.lyx.org/ + https://www.latexproject.org/, mientras que para el de figuras y gráficas hemos utilizado Python+matPlotLib+Pandas+Scipy+Numpy (https://www.scipy.org/). El mapa del libro en el prefacio está confeccionado con Dia https://help.ubuntu.com/ community/Dia.

Este libro debe una parte de su existencia a los proyectos PREDIMED CGL2011-24458 (UIB, Ministerio de Ciencia e Innovación) y COASTEPS CGL201782868-R (UIB, MINECO/AEI/FEDER, UE) en los que participamos.

\section{REFERENCIAS}

SANTOS, C. et al., 2018. Física del caos en la predicción meteorológica. 1156 pp. AEMET. NIPO: 014-18009-X. 